\title{
Performance comparison of multi-wavelength conversion using SOA-MZI and DSF for optical wavelength multicast
}

\author{
Jorge del Val Puente ${ }^{1,2}$, Ni Yan ${ }^{1}$, Eduward Tangdiongga ${ }^{1}$, Ton Koonen ${ }^{1}$ \\ ${ }^{1}$ Electro-optical communication group, Eindhoven University of Technology, Eindhoven, \\ Netherlands. \\ ${ }^{2}$ Grupo de Comunicaciones Ópticas, Universidad de Valladolid, Valladolid, Spain. \\ j.del.val.puente@student.tue.nl
}

\begin{abstract}
The electronic layer multicast is going to face the speed and capacity bottleneck of the future optical data networks. Transparent optical wavelength multicast by multi-wavelength conversion is an effective way of achieving data multicast in the optical domain without any optical-electronic-optical conversion. In this paper, two multiple wavelength conversion technologies for $10 \mathrm{~Gb} / \mathrm{s}$ data rate are investigating and discussed. The first technology is based on cross-phase modulation in a semiconductor optical amplifier - MachZehnder interferometer, and the second is based on four-wave mixing in a dispersion-shifted fiber. We present the simulated performance comparison of two approaches obtained using VPItransmissionMaker ${ }^{T M} W D M$ simulator. Afterwards, we analyze these results in comparison with our previous experimental results of the same schemes.
\end{abstract}

Keywords: Multi-wavelength conversion, multicast, SOA-MZI, FWM, DSF.

\section{Introduction}

In the last few years, optical layer multicasting development has been continuous. Data multicast at the optical layer avoids the needs for Optical-Electronic-Optic (OEO) conversion, and thus can improve the transparency, efficiency and effectiveness of the optical networks [1]. Moreover, it provides other benefits such as simplification of network layer protocols and optical network designs. Multiwavelength conversion (MWC) is a simple way to realize optical wavelength multicast [2]. MWC is very attractive because it can potentially reduce the number of converters in a routing node without adding more complexity in the switch design. Thus switches can be easily adapted for optical layer multicasting [3].

Recently, several methods for MWC have been reported and demonstrated. The MWC and multicasting approaches that are studied in this paper are based in semiconductor optical amplifier - Mach-Zehnder interferometer (SOA-MZI) [4], and in four-wave mixing (FWM) in a dispersion-shifted fiber (DSF). Other methods are based in FWM in a SOA [5], in XGM in a SOA [6], electroabsorption modulator (EAM), etc. Each MWC technique has advantages and disadvantages, and all of them 
have to be studied to know which are more suitable to be adopted in optical switches [7]. This study is normally carried out in laboratories by experimental performances. After that, it is necessary to simulate in a computer the same schemes that have been studied in the laboratory. By this way, we can know if our simulation models developed are correct or not and if the behavior of the devices are similar in the simulations and in the experiments.

In this paper, two MWM aproaches are discussed and compared. Furthermore, simulations of these MWC techniques using $10 \mathrm{~Gb} / \mathrm{s}$ data rate are presented. VPItransmissionMaker ${ }^{T M}$ WDM simulator was used to create the setups of each MWC technique and to make the required simulations. To summarize, this simulation results are compared with the experimental results reported in [7], where experimental performance validation of four MWC techniques was demonstrated.

\section{MWC simulation model}

Simultaneous single-to-multiple-channel wavelength conversions have been simulated with two methods at $10 \mathrm{~Gb} / \mathrm{s}$ bit rate, to evaluate if they are suitable for multicasting. These two techniques are FWM in a DSF and cross phase modulation (XPM) in an SOA-MZI. These two techniques were chosen because, according to our experimental experience, these two schemes were the most promising MWC approaches we have worked with. We also have worked with FWM in a SOA and cross gain modulation in a SOA but the results were not as satisfactory.

The general schematic for these two MWC simulations are shown in Fig.1 [7]. To compare the simulation results with the experiments, we adopted the same general schemes. In all the simulations, four continuous wave (CW) lasers were applied the wavelength probes. Another laser was encoded with $2^{23}-1$ pseudorandom bit sequence by an intensity modulator to obtain the $10 \mathrm{~Gb} / \mathrm{s} \mathrm{NRZ}$ data signal. After EDFA amplification, out-of-band ASE filtering and polarization control, the data signal was injected into the MWC medium. This medium was a SOA-MZI or a DSF. Couplers and a multiplexer were employed to combine data signal and CWs. At the output of the MWC medium, the signal spectrum is monitored and each converted wavelength channel was selected through an optical filter, amplified by another EDFA and the bit-error rate (BER) was measured [7].

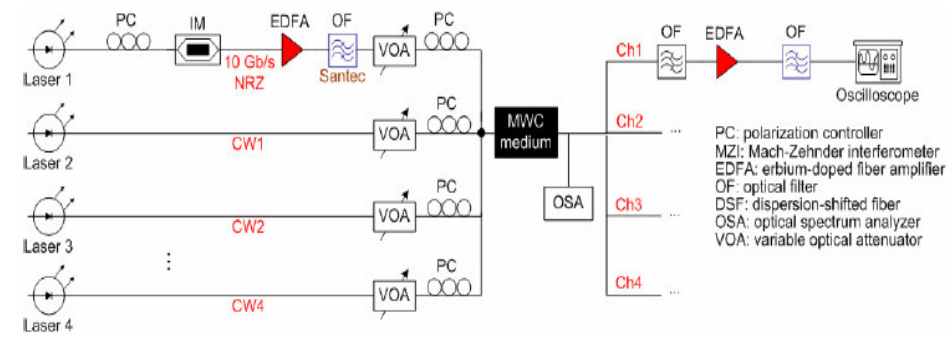

Fig. 1. General schematic for the two MWC simulations. 


\subsection{MWC by SOA-MZI}

SOA-MZI-based MWCs has been reported with good performance [1], [4]. SOA-MZI for MWC has the advantages of compactness and integration probability [4]. High data rate wavelength conversion of $10 \mathrm{~Gb} / \mathrm{s}$ or more generally requires SOA-MZIs which can operate at the same speed, or more complicated setups such as differential scheme have to be employed. In this section, simulation results of one-to-four MWC with $200 \mathrm{GHz}$ and $100 \mathrm{GHz}$ channel spacing (distance between the wavelength multicast channels) are presented.

In the simulation, the signal channel wavelength was set to be $1541.35 \mathrm{~nm} .400$ $\mathrm{GHz}$ detuning was applied to the closest $\mathrm{CW}$ channel. Data signal power before the SOA-MZI was $-1 \mathrm{dBm}$ and the total $\mathrm{CW}$ probe power was $0 \mathrm{dBm}$. Other parameters of the SOA-MZI are summarized in Table 1. The schematic for the SOA-MZI MWC is shown in Fig 2.

Table 1. Parameters for the SOA-MZI.

\begin{tabular}{lcc}
\hline \multicolumn{1}{c}{ Parameter name } & Value & Units \\
\hline \multicolumn{1}{c}{$\begin{array}{c}\text { Injection Current SOA 1 } \\
\text { 200 GHz Channel Spacing }\end{array}$} & 0.35 & $\mathrm{~A}$ \\
100 & 0.313 & $\mathrm{~A}$ \\
Injection Current SOA 2 & & \\
\multicolumn{1}{c}{ 200 GHz Channel Spacing } & 0.281 & $\mathrm{~A}$ \\
SOA Length & 1 & $\mathrm{Am}$ \\
SOA Width & 1 & $\mu \mathrm{m}$ \\
SOA Height & 0.2 & $\mu \mathrm{m}$ \\
Optical Confinement & 0.3 & \\
Internal Losses & 3000 & $1 / \mathrm{m}$ \\
Differential Gain & $2.8 \mathrm{e}-20$ & $\mathrm{~m}$ \\
Carrier Density at Transparency & $1.4 \mathrm{e} 24$ & $1 / \mathrm{m}^{3}$ \\
IndexToGainCoupling & 3 & \\
Linear Recombination Constant (A) & $1.43 \mathrm{e} 8$ & $1 / \mathrm{s}$ \\
Bimolecular Recombination Constant (B) & $1.0 \mathrm{e}-16$ & $\mathrm{~m}^{3} / \mathrm{s}$ \\
Auger Recombination Constant (C) & $1.3 \mathrm{e}-41$ & $\mathrm{~m}^{6} / \mathrm{s}$ \\
Initial Carrier Density & $2.0 \mathrm{e} 24$ & $1 / \mathrm{m}^{3}$ \\
\hline
\end{tabular}

$200 \mathrm{GHz}$ channel spacing MWC. The output spectrum is illustrated in Fig. 3. The eye diagrams of each wavelength-converted channel are represented in Fig. 4. All output channels had clear open eyes. BER measurements of the back-to-back signal (b2b) and converted channels are shown in Fig.5, from which we observe around 0.4 $\mathrm{dB}$ power penalty for the copies at BER equal to $10^{-9}$. Besides, eye extinction ratio (ER) of the converted signals measured is around $29,5 \mathrm{~dB}$ in each channel. 


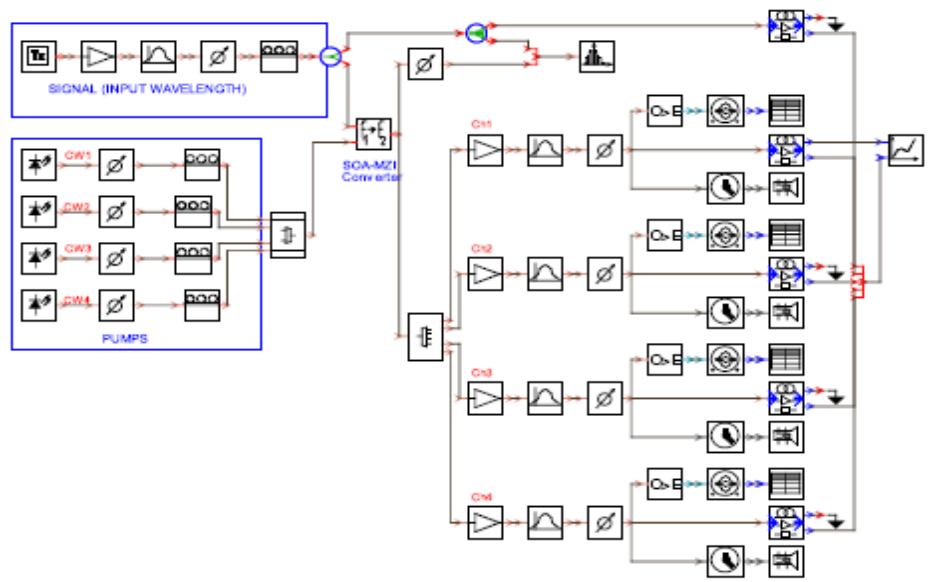

Fig. 2. Schematic for the SOA-MZI MWC

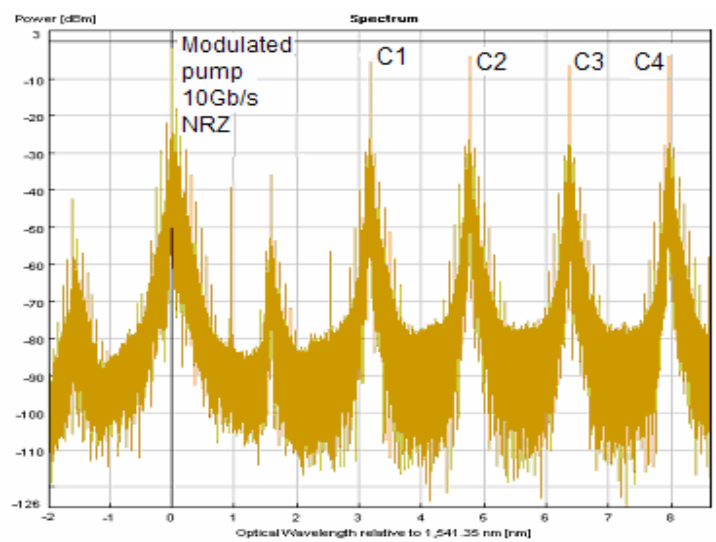

Fig. 3. SOA-MZI MWC: $200 \mathrm{GHz}$ channel spacing Spectrum
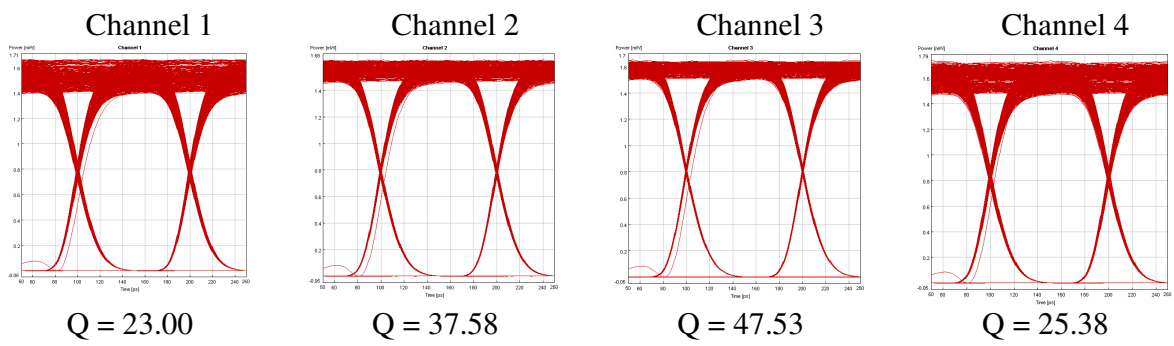

Fig. 4. SOA-MZI MWC: $200 \mathrm{GHz}$ channel spacing converted eye diagrams 


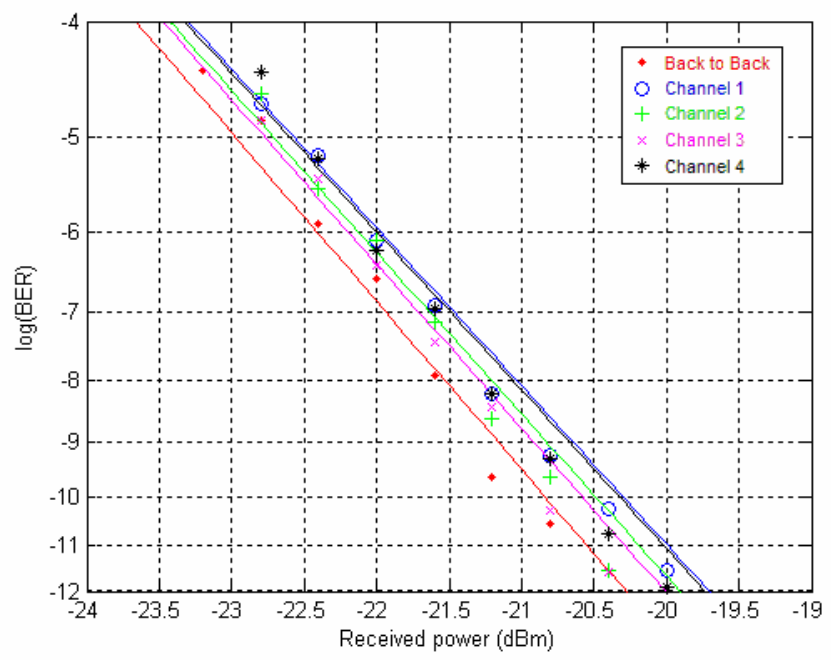

Fig. 5. SOA-MZI MWC: $200 \mathrm{GHz}$ channel spacing BER measurements

100 GHz channel spacing MWC. The results are shown in Fig. 6, Fig. 7 and Fig. 8. In this case, the eyes were less opened than in previous one. This was because channels were nearer now and the crosstalk between them was bigger. Thus, the simulated Q factors were also lower (from 10.5 in the first channel, to 14.4 in the second one) comparing to that of the $200 \mathrm{GHz}$ channel spacing MWC. From the spectrum we also observe more satellite products generated. Also from the BER curve we observe an error floor for channel 1 and 4 . The power penalty at $10^{-9}$ increased to about $3 \mathrm{~dB}$. Finally, eye ER is reduced to around $12.6 \mathrm{~dB}$ in this simulation.

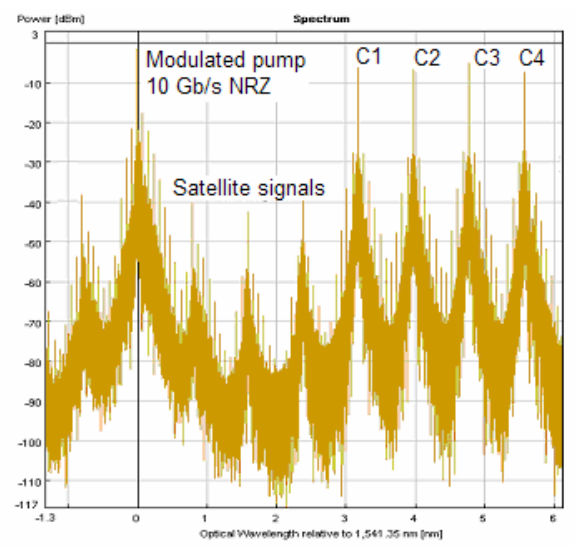

Fig. 6. SOA-MZI MWC: $100 \mathrm{GHz}$ channel spacing Spectrum 

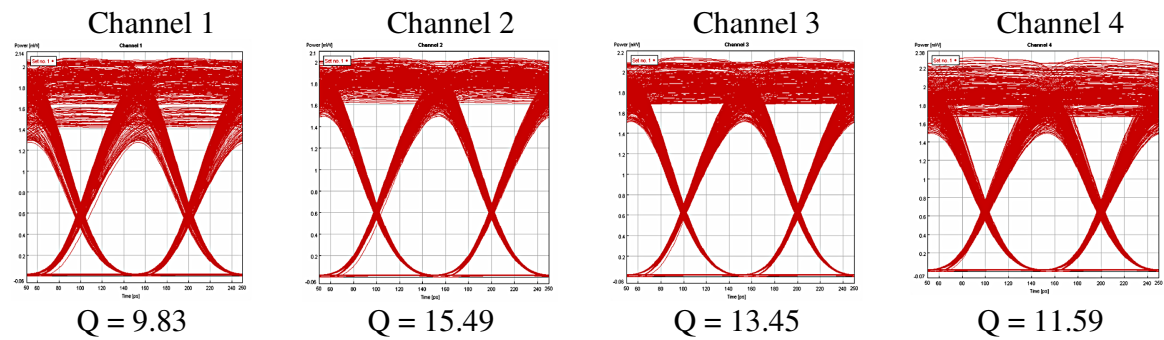

Fig.7. SOA-MZI MWC: $100 \mathrm{GHz}$ channel spacing converted eye diagrams

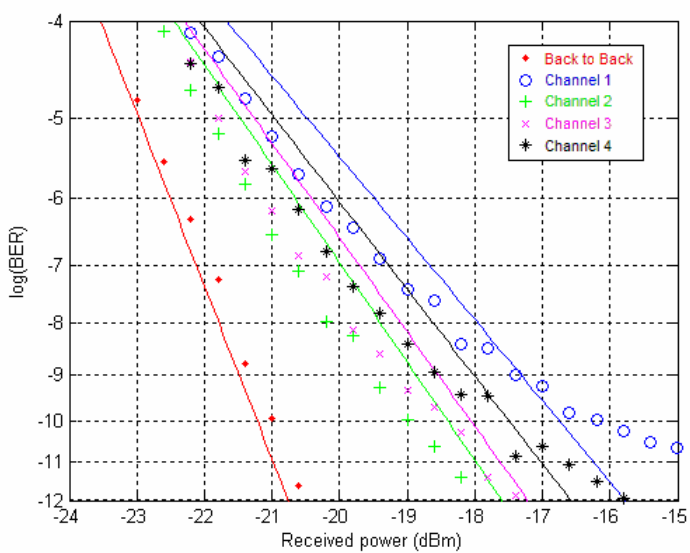

Fig. 8. SOA-MZI Wavelength Converter: $100 \mathrm{GHz}$ channel spacing BER measurements

The much worse results that we got from bring the channels closer were due to the increased inter-channel crosstalk, though at $100 \mathrm{GHz}$ spacing we still got open eyes and reasonably good performance. In latter case, channel 2 had the shortest power penalty, while with the former channel spacing channel 3 had the shortest power penalty.

\subsection{MWC by FWM in dispersion-shifted fiber (DSF)}

In principle, FWM for wavelength conversion offers strict transparency in both bit rate and modulation format. Moreover, in theory FWM in DSF has no limitations in relation to bit rate or operation speed.

In this MWC schematic, data signal was placed at $1547.72 \mathrm{~nm}$, the zero-dispersion wavelength of the DSF, and four other tunable lasers were used as CWs (continuous waves). The laser was encoded with $2^{23}-1$ bits by a MZI in order to form the $10 \mathrm{~Gb} / \mathrm{s}$ pseudorandom pump NRZ data. $200 \mathrm{GHz}$ detuning and $100 \mathrm{GHz}$ channel spacing were used. The schematic for the FWM in DSF MWC is shown in Fig 9. 
The output signal power was set to $12 \mathrm{dBm}$, pump average powers were $0 \mathrm{dBm}$ each. Just before the SOA, signal power was $9.61 \mathrm{dBm}$ and the total $\mathrm{CW}$ power was 0 $\mathrm{dBm}$. Pump signal here needed much higher power than that in the SOA-MZI MWC setup.

In this case, polarization controllers were very important because FWM in DSF was polarization sensible.

The simulated output spectrum is illustrated in Fig. 10. The measured average MWC conversion efficiency was around $-18 \mathrm{~dB}$. Eye diagrams are shown in Fig. 11 . It is noticeable that all the eyes had a quite good opening, but they had more noise that in SOA-MZI case. Q factor values were good (from 9.2 in the first channel, to 14.3 in the last one). BER measurements of the back-to-back signal (b2b) and converted channels are shown in Fig.12. The output ER of our scheme is near $23 \mathrm{~dB}$ in each converted channel. In this case, at BER of $10^{-9}$ the power penalty was nearly 0 $\mathrm{dB}$ for the third and forth channels, about $0.3 \mathrm{~dB}$ for the second one, and about $0.8 \mathrm{~dB}$ for the first channel.

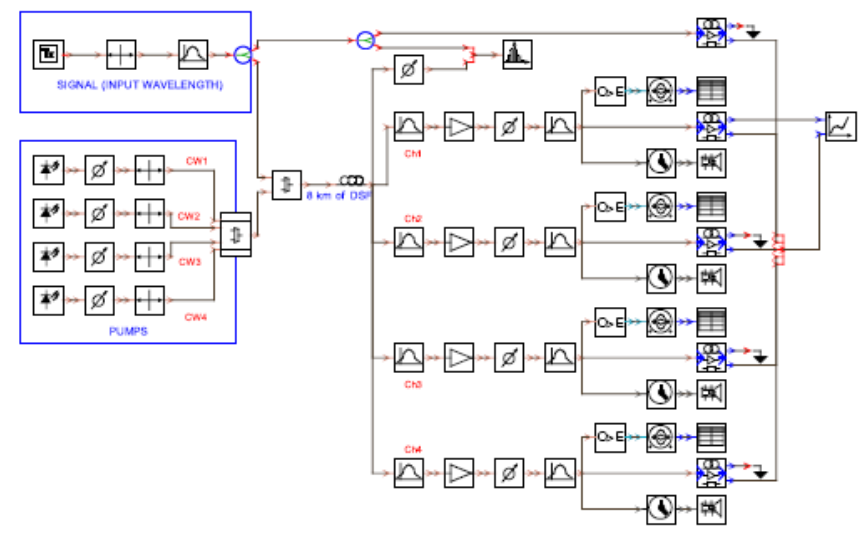

Fig. 9. Schematic for the FWM in DSF MWC

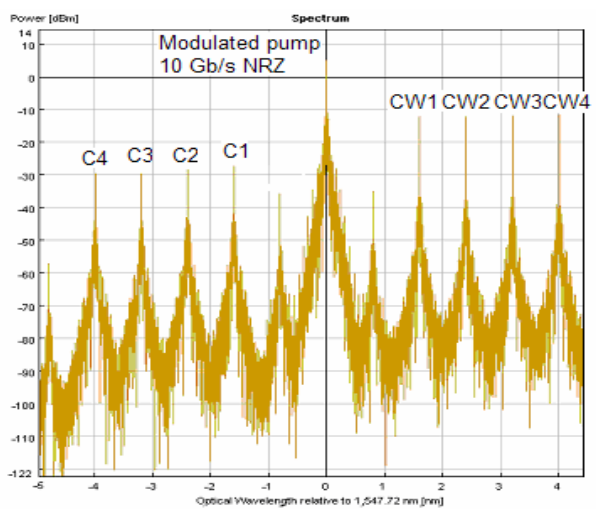

Fig. 10. FWM Wavelength Converter: Spectrum at DSF output 

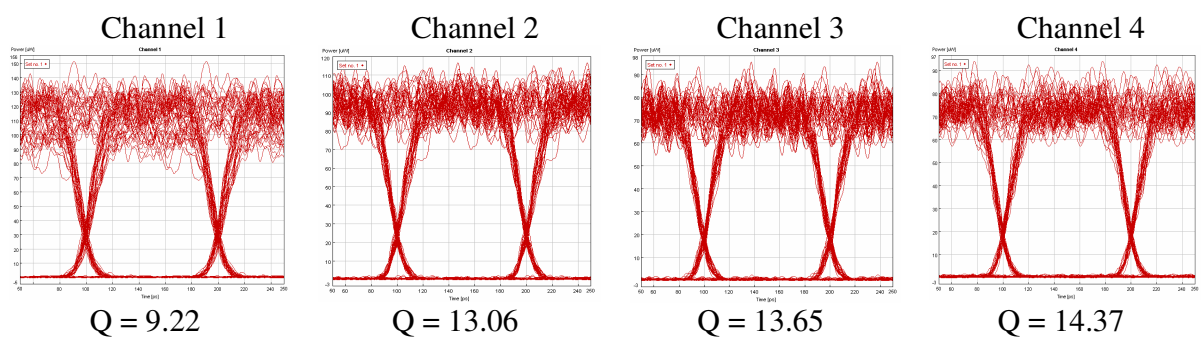

Fig. 11. FWM Wavelength Converter in DSF: converted eye diagrams

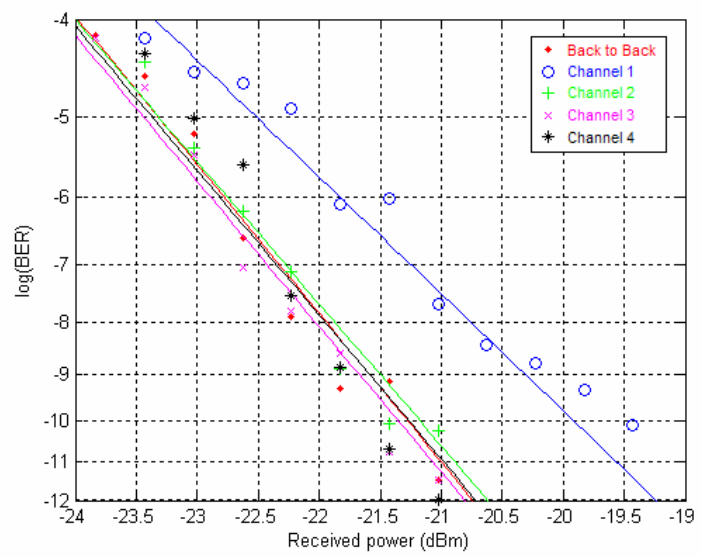

Fig. 12. FWM Wavelength Converter in DSF: BER measurements

\subsection{Comparison of SOA-MZI and FWM in DSF MWCs}

Both SOA-MZI and DSF-based multi-wavelength converters have their advantages and disadvantages. FWM in DSF needs the modulated pump signal to be placed around the fiber zero-dispersion wavelength, which restricted the wavelength flexibility of the scheme. Using SOA-MZI for MWC, the modulated signal can be placed on any wavelength within both SOAs' gain spectra. Besides, SOA-MZI setup needs less input power than the other.

All the eye diagrams obtained in both schemes have clear opening and noise suppression at the zero level. However the quadratic effect to the signal power also amplified the noise at logical 'one' level and caused signal distortion at this level. Q factor from the simulation result are quite good: higher than 9 in FWM in DSF scheme, higher than 10.5 in SOA-MZI with $100 \mathrm{GHz}$ channel spacing and higher in SOA-MZI with $200 \mathrm{GHz}$ spacing. Because of the simulation environment, these 
results cannot give absolute credit for comparing the MWC scheme in real, but it helps us to a certain extend to understand the important parameters that matters for each scheme stand alone.

FWM in DSF has the least power penalties and excellent eye ER measurements. These results are better than SOA-MZI results with the same channel spacing (100 $\mathrm{GHz}$ ). Besides, FWM in DSF detuning is half of the other scheme detuning and crosstalk between channels should be higher.

\section{Comparison of simulation and experimental results}

Simulation results shown in the previous section are largely coherent to experimental results shown in [7]. However simulation provides more convenience in changing the parameters and assesses their importance to the scheme.

Comparing to the SOA-MZI MWC experimental results, with $200 \mathrm{GHz}$ channel spacing, obtained power penalty was around $0.5 \mathrm{~dB}$, while in the simulations it was around $0.7 \mathrm{~dB}$. In both the simulations and experiments, every converted channel had clear open eyes, but in experiments, the eye 'zero' level has more noise than what was shown in simulations. It might due to the fact that the simulator neglected some effects that added noises at 'zero' level. In experiments, converted signals Q factor values were also quite good (around 10-11), which was confirmed by the simulation results.

With $100 \mathrm{GHz}$ channel spacing SOA-MZI MWC, experiments achieved much less power penalty than in the simulations (less than $0.5 \mathrm{~dB}$ in experiments, and around $3 \mathrm{~dB}$ in simulations). Both simulation and experimental results had similar eye opening diagrams (more noisy in the experiments) and Q factor (near 10).

Experimental results in FWM in DSF MWC scheme were as good as simulation ones: similar eye diagrams with a noisy 'one' level, conversion efficiency around -15 $\mathrm{dB}(-13.5 \mathrm{~dB}$ in experiments and $-18 \mathrm{~dB}$ in simulations), small power penalties and $\mathrm{Q}$ factor values around 11 .

\section{Conclusions}

For MWC at bit rate below 10-40 Gb/s, SOA-MZI-based wavelength converter can be a very good choice. This technique has an excellent compactness and conversion flexibility in wavelengths. It also requires very low optical input power, limiting a possible high crosstalk between channels. Both simulations and our previous experiments show that this scheme produces MWC with low power penalty between the converted channels and the back-to-back signal at $200 \mathrm{GHz}$ channel spacing. Besides, converted channels have quite good eye opening and Q factor values.

On the other hand, FWM in DSF offers transparency in data rate and modulation format, which can be suitable for very high bit rate operation towards $\mathrm{Tb} / \mathrm{s}$ range. In this case, the number of converted channels has little impact on conversion efficiency and converted $\mathrm{Q}$ factor as long as they are placed within the effective conversion 
detuning range from the modulated signal pump. However, this setup needs high optical input power and is very sensitive to the polarization of all the channels.

Finally, our simulation results and previous experimental results showed similar behavior, which confirmed the correctness of our simulation models developed. Some slight difference is due to the fact that it was impossible to emulate the real experimental environment, and the component models provided in the simulator cannot be completely adjust to present the exact devices we used.

Acknowledgments. This work has been supported by the European Commission through the Network of Excellence e-Photon/ONe+ project. The author would like to thank the IST-LASAGNE project for funding these research activities, and Dr. Javier Herrera Llorente for his help with the simulator.

\section{References}

1. N. Yan, et al: Simultaneous one-to-four multi-wavelength conversion using a single SOAMZI, 11th European Conference on Networks and Optical Communications (NOC), 2006.

2. F. Ramos et al., IST-LASAGNE: Towards All-Optical Label Swapping Employing Optical Logic Gates and Optical Flip-flops, IEEE Journal of Lightwave Technology, October 2005.

3. N. Yan, A. Teixeira et al.: Simultaneous multi-wavelength signal conversion for transparent optical multicast. European Conference on Networks Optical communications (NOC), 2005

4. H. S. Chung et al.: All-optical multi-wavelength conversion of $10 \mathrm{Gbit} / \mathrm{s}$ NRZ/RZ signals based on SOA-MZI for WDM multicasting. Electron. Lett., Vol. 41, no.7, Mar. 2005.

5. S. Diez, C. Schmidt et al.: Four-Wave Mixing in Semiconductor Optical Amplifiers for Frequency Conversion and Fast Optical Switching, IEEE Journal of selected topics in Quantum Electronics, Vol. 3, No. 5, October 1997.

6. T.G. Silveira, et al.: Cross-gain modulation bandwidth enhacement in semiconductor optical amplifiers by means of detuned optical filter, IEEE Electronics Letters, Vol.41, no. 13, 23rd June 2005.

7. N. Yan, A. Teixeira, T. Silveira, I. Tafur Monroy, H.-D. Jung, T. Koonen, Optical multicast technologies by multi-wavelength conversion for optical routers, Proc. IEEE 2006 10th International Conf. on Communication Technology (ICCT'2006), 27-30 Nov 2006, Guilin, China, paper SRGN (\#2006072901). 